The Journal of Laryngology \& Otology

http://journals.cambridge.org/JLO

Additional services for The Journal of Laryngology \& Otology:

Email alerts: $\underline{\text { Click here }}$

Subscriptions: $\underline{\text { Click here }}$

Commercial reprints: $\underline{\text { Click here }}$

Terms of use : $\underline{\text { Click here }}$

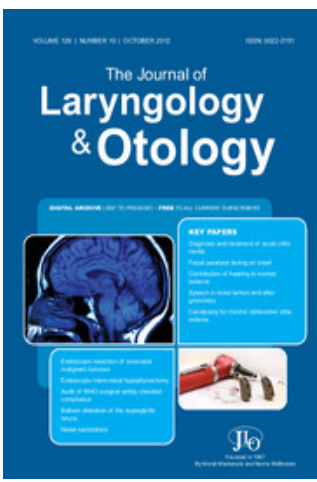

\title{
The use of clostridium botulinum toxin in palatal myclonus. A preliminary report
}

Shakeel R. Saeed and Gerald B. Brookes

The Journal of Laryngology \& Otology / Volume 107 / Issue 03 / March 1993, pp 208 - 210

DOI: 10.1017/S0022215100122650, Published online: 29 June 2007

Link to this article: http://journals.cambridge.org/abstract_S0022215100122650

How to cite this article:

Shakeel R. Saeed and Gerald B. Brookes (1993). The use of clostridium botulinum toxin in palatal myclonus. A preliminary report. The Journal of Laryngology \& Otology, 107, pp 208-210 doi:10.1017/S0022215100122650

Request Permissions : $\underline{\text { Click here }}$ 


\title{
The use of clostridium botulinum toxin in palatal myoclonus. A preliminary report
}

\author{
Shakeel R. Saeed F.R.C.S., Gerald B. Brookes F.R.C.S. (London)
}

\begin{abstract}
Palatal myoclonus is a rare syndrome characterized by involuntary rhythmical movements of the soft palate giving rise to clicking objective tinnitus. The intrusive nature of the tinnitus prompts patients to seek medical advice but to date no single treatment modality has been shown to be consistently effective. We present three cases in whom various management regimes were unsuccessful and in whom botulinum toxin injection to the palatal muscles was undertaken. All three cases were rendered free of their tinnitus with complete abolition of the myoclonus. The questions of optimum dosage as well as frequency of injection will be answered as greater numbers are treated by this method.
\end{abstract}

Key words: Clostridium botulinum; Myoclonus, palatal

\section{Introduction}

The tinnitus associated with palatal myoclonus has been traditionally managed with drugs such as anxiolytics, antidepressants and anticonvulsants or surgical procedures aimed at modifying palatal and eustachian tube function as well as adjunctive measures such as psychotherapy. The tinnitus however is often refractory to these measures though more recently white-noise masking has shown more favourable results in selected cases. We describe an alternative treatment modality aimed at abolishing the myoclonic activity by selective injection of the palatal muscles with botulinum toxin.

\section{Case reports}

\section{Case 1}

A 25-year-old male presented initially with right-sided headaches, blurred vision, episodic dysphagia and clicking in both ears. General and neurological examination was unremarkable and investigations including computerized tomography of the head were normal. Following psychiatric assessment and treatment with an anxiolytic drug the patient's headaches resolved.

The tinnitus however persisted and otological appraisal confirmed an audible clicking noise emanating from the right side of the face. Flexible nasendoscopy with topical anaesthesia showed bilateral myoclonic activity of the soft palate synchronous with the audible bruit. Pure tone audiometry and tympanometry were normal and having explained the findings to the patient five mouse-units of botulinum toxin were injected into both sides of the soft palate. Outpatient review at four and twelve weeks showed the patient to be free of his tinnitus with no untoward effects.

\section{Case 2}

A 64-year-old female presented for consideration of botulinum toxin injection for palatal myoclonus. She had suffered intractable clicking noises in her head for twelve years that were often of sufficient intensity to be heard by her husband. The original somewhat speculative diagnosis of minor brainstem vascular accident had been managed with benzodiazepines. The tinnitus was refractory to treatment with various antidepressants and anticonvulsants as well as physiotherapy and relaxation exercises.

On examination the patient had easily audible tinnitus in time with the visible rhythmical movements of the soft palate. Five mouse-units of botulinum toxin were injected into the soft palate bilaterally and the myoclonus abated for a few hours. Repeated injections on two subsequent occasions had a similar effect but a fourth injection of fifteen mouse-units resulted in marked nasal regurgitation and dysphagia with minimal improvement in the tinnitus. The adverse effects resolved completely in four weeks but the tinnitus persisted.

Three months later the patient requested a further trial of injection therapy and gained a marked improvement in her tinnitus with no side-effects following injection of ten mouse-units of the toxin into the palate bilaterally.

\section{Case 3}

A 70-year-old female had suffered with palatal myoclonus and intrusive tinnitus for seven years. Initial investigations including cerebrocervical angiography had been negative. Antidepressants and anxiolytic drugs were unhelpful and indeed the only medication that had given any relief was carbamazepine but unfortunately the patient developed a cutaneous sensitivity reaction neces- 
sitating withdrawal of the drug. Subsequent treatment with white-noise masking, eustachian tube diathermy and even hamulectomy had been unfruitful. On this basis the patient was referred for botulinum toxin injection and ten mouse-units were injected bilaterally into the palatal muscles.

The patient reported some initial difficulty with swallowing and speech secondary to palatal weakness but the myoclonus and the associated tinnitus were abolished. This state of remission was maintained for one year and repeat injection with five mouse-units bilaterally allowed the patient to remain free of the myoclonus.

\section{Discussion}

Palatal myoclonus is a rare but increasingly reported syndrome characterized by involuntary rhythmical movements of the soft palate, usually bilaterally, with clonic rates of 40 to 240 per minute. The condition was first reported in 1873 by Kupper though Spencer coined the phrase 'pharyngeal and laryngeal mystagmus' (Spencer, 1886). The objective clicking tinnitus associated with the myoclonic contractions had already been noted by Politzer in 1887 who postulated that the snapping shut of the eustachian tube following tubal opening by the actions of the palatine muscles gave rise to the clicking noises (Samant et al., 1970). The condition is considered as a disturbance of cerebello-medullary neural function and may be associated with myoclonic activity in the pharyngeal, laryngeal, ocular and diaphragmatic muscles. Cases have been observed following blunt and penetrating trauma to the head and neck, infective inflammatory conditions such as viral encephalitis, malaria and syphilis, demyelinating diseases, aneurysms of the vertebral artery, posterior fossa tumours as well as in heredofamilial tremor. Seventy per cent of cases however are secondary to vascular infarcts (Fitzgerald, 1984). Whilst no specific nervous pathway or nuclear lesion has been definitively implicated various studies have traced the pathogenesis to an area consisting of the dentate nucleus, red nucleus and the inferior olivary nucleus, collectively constituting the Guillain-Mollaret triangle (Siegel, 1987). Further refinements have demonstrated hypertrophic degeneration of the contralateral inferior olivary nucleus, the whole area being referred to as the myoclonic triangle (Lapresle, 1979). Experimental injury to the inferior olive in monkeys has produced the characteristic myoclonus adding further evidence to this neurological localization (Fitzgerald, 1984).

Investigation of patients presenting with palatal myoclonus aims at excluding causes such as those cited earlier before terming a case as idiopathic. In particular, pure tone and evoked response audiometry with tympanometry to exclude middle and inner ear disorders as well as magnetic resonance imaging of the posterior fossa and brainstem should be undertaken.

Numerous treatment modalities have been used in an attempt to abolish the often incapacitating tinnitus that these patients suffer. None has been shown conclusively to confer lasting benefit. Therapeutic regimes including anxiolytic, antidepressant and anticonvulsant drugs have yielded varying results as have physiotherapy, acupuncture, relaxation exercises and hypnosis. Attempts at topical and infiltrative local anaesthesia have been unfruitful as have surgical procedures such as palatal muscle section and tenotomy of the muscles of the middle ear cleft. More recently, the results of white-noise masking in selected cases have been encouraging, indeed eleven out of twelve patients treated this way gained some benefit with three patients being rendered symptom free. The source of the actual clicking is postulated to arise from the breakdown of fluid surface tension as the walls of the eustachian tube open under the action of the peritubal muscles (East and Hazell, 1987). The myoclonus however is characteristically resistant to current methods of treatment and we considered that local botulinum toxin injections may confer benefit.

Clostridium botulinum toxin is a protein which, if injected into a muscle selectively weakens it. The toxin binds to the motor end-plate and prevents acetylcholine release giving rise to pre-synaptic neuromuscular blockade. This leads to atrophy and weakness of the muscle which can last between two to twenty days though recovery by formation of new terminal axons can take up to four months. The actual doses used are related to the muscle bulk to be weakened and further injections are titrated against the response (Moore, 1991). The drug is a noninfectious toxin-haemagglutinin complex and whilst six toxin serotypes have been isolated only type $\mathrm{A}$ is in clinical use. The potency is standardised in mouse $\mathrm{LD}_{50}$ units

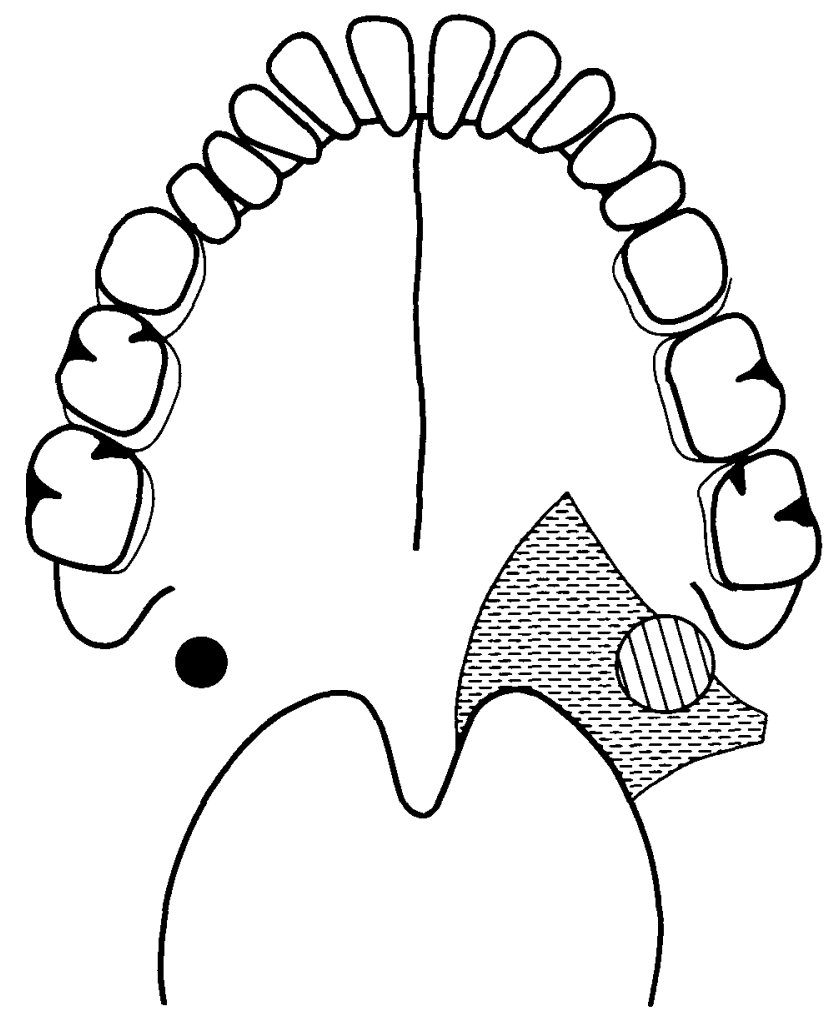

Site of injection

Q Insertion of Levator Veli Palatini

$\theta$ Insertion and aponeurosis of Tensor Veli Palatini

Fig. 1

Schematic diagram to show the site of injection of the botulinum toxin. 
with 40 mouse-units corresponding to $1 \mathrm{ng}$ of UK toxinhaemagglutinin complex. (Mountain et al., 1992). The drug already has an established role in the management of hemifacial spasm and focal blepharospasm for which it is licenced and is being increasingly used for spasmodic torticollis, strabismus and oromandibular dystonias. Our department has considerable clinical experience in the treatment of both adductor and abductor spasmodic laryngeal dystonias using this agent with good long term results. Side-effects are generally minor and attributable to transient weakness of muscles affected by local spread of the toxin. Usage in children has not been evaluated (Marsden and Quinn, 1990).

To our knowledge botulinum toxin has not been used previously in the treatment of palatal myoclonus. The three cases described illustrate how current treatment modalities can be exhausted leaving the patient with intractable intrusive tinnitus. The patients were offered toxin injection after having explained the potential benefits and inherent side-effects of such treatment. The drug is injected posteromedial to the maxillary tuberosity in an attempt to paralyse the levator and tensor veli palatini muscles which are the prime openers of the eustachian tube (Fig. 1). Two of the cases gained marked benefit from the treatment with abolition of the myoclonus for several months. Case 2 however required repeated injections and illustrated that one cannot invariably expect a favourable outcome and indeed the local side-effects may initially detract from continuing treatment. Further evaluation is required, in particular to establish optimal doses and injection frequency and hopefully this information will become available as more patients are treated by this means.

\section{Acknowledgement}

We wish to express our gratitude to the Department of Medical Illustration at the Royal National Throat, Nose and Ear Hospital for their help with the line drawing.

\section{References}

East, C. A., Hazell, J. W. P. (1987) The suppression of palatal (or intra-tympanic) myoclonus by tinnitus masking devices. A preliminary report. Journal of Laryngology and Otology 101: $1230-1234$.

Fitzgerald, D. C. (1984) Palatal myoclonus-case report. Laryngoscope 94: 217-219.

Lapresle, J. (1979) Rhythmic palatal myoclonus and the dentoolivary pathway. Journal of Neurology 220: 223-230.

Marsden, C. D., Quinn, N. P. (1990) The dystonias. British Medical Journal 300: 139-144.

Moore, A. (1991) Botulinum toxin. Prescribers' Journal, 31: 197-202.

Mountain, R. E., Murray, J. A. M., Quaba, A. (1992) Management of facial synkinesis with Clostridium botulinum toxin injection. Clinical Otolaryngology 17: 223-224.

Samant, H. C.. Gupta, S. K., Gupta, O. P. (1970) Palatal myoclonus. Annals of Otology, Rhinology, Laryngology 79: 858-861.

Siegel, M. A. (1987) Bilateral palatal myoclonus: report of case. Journal of American Dental Association 114: 333-334.

Spencer, H. R. (1886) Pharyngeal and laryngeal nystagmus. Lancet 2: $702-704$

Address for correspondence:

Shakeel R. Saeed, F.R.C.S.

University Department of Otolaryngology,

Manchester Royal Infirmary,

Oxford Road,

Manchester,

M13 9WL. 